\title{
Prevalência de fatores de risco e proteção para doenças crônicas nas capitais do Brasil - principais resultados do Vigitel 2010
}

\author{
Protection from chronic diseases and the prevalence of risk factors \\ in Brazilian state capitals - main results from Vigitel 2010
}

Betine Pinto Moehlecke Iser ${ }^{1}$

Renata Tiene de Carvalho Yokota ${ }^{1}$

Naiza Nayla Bandeira de Sá ${ }^{1}$

Lenildo de Moura ${ }^{1}$

Deborah Carvalho Malta ${ }^{1}$

${ }^{1}$ Coordenação Geral de Doenças e Agravos Não Transmissíveis,

Departamento de Análise de Situação de Saúde, Secretaria de Vigilância em Saúde, Ministério da Saúde. SAF Sul, Trechos 02, Lotes 05/06, Bloco F, Torre 1 Edifício Premium, Térreo, Sala 14. 70070-600 Brasília DF. deborah.malta@saude.gov.br
Abstract Purpose: To describe protection from chronic diseases and the prevalence of risk factors with data from a telephone survey in 2010. Methods: Telephone interviews in a random sample of adults living in Brazilian state capitals and the Federal District with residential landline telephones. The prevalence of these factors was stratified by sex, age and level of education. Results: High prevalence of soft drink consumption (28.1\%), fatty meat consumption (34.2\%), and alcohol abuse (18\%) and low fruit and vegetable intake (18\%) and leisure time physical activity (15\%) was verified. Approximately half the population was overweight and reported no sun protection practices. Physical inactivity and smoking afflicted almost 15\% of adults. In general, risk factors were more prevalent in men, predominantly young adults with lower education levels. Conclusion: The results revealed differing health behavior according to socio-demographic variables. These variables should be taken into consideration in health promotion campaigns.

Key words Risk factors, Chronic disease, Epidemiological studies, Surveillance, BRFSS (Behavioral Risk Factor Surveillance System)
Resumo Objetivo: descrever os principais fatores de risco e proteção para doenças crônicas com dados do inquérito telefônico de 2010. Metodologia: entrevistas telefônicas realizadas em amostra probabilística dos adultos residentes nas capitais do Brasil e Distrito Federal com telefone fixo residencial. As prevalências foram estratificadas por sexo, idade e escolaridade. Resultados: Foi verificado alto consumo de refrigerantes (28\%), de carne com gordura (46\%) e de consumo abusivo de álcool (18\%), e baixo consumo de frutas e hortaliças (18\%) e de atividade física no lazer (15\%). Aproximadamente metade da população referiu ter excesso de peso e não se proteger da radiação solar. A inatividade física e o tabagismo atingem cerca de 15\% dos adultos. Fatores de risco foram mais prevalentes em homens, adultos jovens e de menor escolaridade. Conclusão: os resultados apontam diferentes comportamentos em saúde da população segundo características sociodemográficas. Essa variabilidade deve ser considerada nas ações de promoção de saúde.

Palavras-chave Fatores de risco, Doença crônica, Levantamentos epidemiológicos, Vigilância, Estudos transversais 


\section{Introdução}

O crescimento da magnitude das doenças crônicas não transmissíveis (DCNT) na morbimortalidade populacional nos últimos anos gerou uma preocupação mundial em torno do impacto destas doenças na qualidade de vida e desenvolvimento econômico dos países ${ }^{1}$. Em 2011, a Organização das Nações Unidas conclamou os governos e a sociedade mundial a definir estratégias comuns de enfrentamento a essas doenças, com foco nas cardiovasculares, diabetes, respiratórias crônicas e câncer, as quais juntas são responsáveis por cerca de $58 \%$ das mortes e $50 \%$ das incapacidades ocorridas no mundo ${ }^{2,3}$.

No Brasil, essas quatro doenças foram responsáveis por $58 \%$ das mortes ocorridas em 2007 e por cerca de $26 \%$ das internações hospitalares não relacionadas a gestações registradas em $2009^{4,5}$. Em consonância com a preocupação mundial, o Brasil elaborou em 2011 um Plano de Ações Estratégicas Para o Enfrentamento das Doenças Crônicas Não Transmissíveis (DCNT) no Brasil para os próximos dez anos, com a participação de diferentes setores do governo e da sociedade civil ${ }^{6}$.

Além de responsáveis pela alta carga das DCNT, outra característica comum a essas quatro doenças são seus fatores de risco modificáveis, quais sejam: tabagismo, álcool, inatividade física, alimentação não saudável e obesidade ${ }^{7}$, reconhecidamente passíveis de prevenção a partir de estratégias comuns de melhoria dos hábitos de saúde da população, e por isso destacados no plano mencionado.

No Brasil, o sistema de Vigilância de Fatores de Risco e Protecão para doenças crônicas por inquérito telefônico, o Vigitel, permite o monitoramento destes e de outros fatores de risco e proteção ao desenvolvimento de doenças crônicas. A partir da implantação desse sistema, em 2006, a vigilância passou a dispor de informações a respeito da distribuição desses fatores na população adulta residente nas capitais do país, embasando a elaboração de políticas de saúde específicas, de acordo com a situação de saúde da população ${ }^{8}$.

O presente artigo descreve os principais resultados do inquérito Vigitel no ano de 2010, apresentando as prevalências dos fatores de risco e proteção estudados, segundo sexo, idade e escolaridade da população adulta das capitais do Brasil.

\section{Métodos}

Foram utilizados os dados do Sistema de Vigilância de Fatores de Risco e Proteção para Doenças Crônicas - Vigitel, coletados por meio de entrevistas telefônicas realizadas de janeiro a dezembro de 2010. O Vigitel é um estudo transversal, representativo para a população adulta ( $>$ 18 anos) residente nas 26 capitais do Brasil e no Distrito Federal, realizado anual e continuamente pelo Ministério da Saúde desde 2006. Em 2010, foram realizadas cerca de 2000 entrevistas telefônicas em cada um dos 27 entes federados.

O Vigitel utiliza amostras probabilísticas da população com 18 anos ou mais residente em domicílios servidos por pelo menos uma linha fixa de telefone, obtidas em dois estágios: 1) sorteio sistemático de 5.000 linhas telefônicas em cada cidade, seguido por ressorteio e organização de 25 subasmostras de 200 linhas, sendo consideradas elegíveis para o sistema as residenciais e ativas. 2) sorteio de um morador adulto do domicílio para responder a entrevista.

A entrevista do VIGITEL se baseia no sistema ETAC (entrevistas telefônicas assistidas por computador), onde as perguntas são lidas na tela do computador e suas respectivas respostas registradas diretamente em meio eletrônico, permitindo pulos automáticos de questões não válidas decorrentes das respostas anteriores e minimizando erros de digitação. Maiores detalhes sobre o desenho amostral e procedimentos metodológicos encontram-se publicados ${ }^{8,9}$.

Neste estudo são descritas as frequências dos principais indicadores relacionados a doenças crônicas em adultos, no conjunto das capitais brasileiras e no Distrito Federal, no ano de 2010. Os fatores de risco apresentados incluem: tabagismo; excesso de peso (Índice de Massa Corpo$\mathrm{ral}>25 \mathrm{~kg} / \mathrm{m}^{2}$ ); obesidade (Índice de Massa Corporal $>30 \mathrm{~kg} / \mathrm{m}^{2}$ ); consumo de carnes com excesso de gordura (carne vermelha com gordura visível ou frango com pele); consumo regular (cinco ou mais dias por semana) de refrigerantes ou suco artificial; inatividade física (indivíduos que não praticaram qualquer atividade física no tempo livre nos últimos três meses, que não realizam esforços físicos intensos no trabalho, que não se deslocam para o trabalho ou escola a pé ou de bicicleta e que não são responsáveis pela limpeza pesada de suas casas); consumo abusivo de bebidas alcoólicas (quatro ou mais doses para mulher e cinco ou mais doses para homem 
em uma mesma ocasião nos últimos 30 dias, considerando como dose de bebida alcoólica uma dose de bebida destilada, uma lata de cerveja ou uma taça de vinho); autoavaliação do estado de saúde ruim; e morbidades referidas (diagnóstico médico prévio de hipertensão arterial, diabetes e asma/bronquite crônica/enfisema).

Os fatores de proteção avaliados foram: consumo recomendado (cinco ou mais porções diárias, em cinco ou mais dias da semana) de frutas e hortaliças; consumo regular (cinco ou mais dias da semana) de feijão; atividade física no lazer (prática de atividade de intensidade leve ou moderada - caminhada, caminhada em esteira, musculação, hidroginástica, ginástica em geral, natação, artes marciais e luta, bicicleta, voleibol ou outra - por pelo menos 30 minutos diários em cinco ou mais dias da semana ou atividades de intensidade vigorosa - corrida, corrida em esteira, ginástica aeróbica, futebol, basquetebol ou tênis - por pelo menos 20 minutos diários em três ou mais dias da semana); proteção contra radiação solar (uso de proteção solar quando exposto ao sol por mais de 30 minutos, seja andando na rua, no trabalho ou no lazer).

As estimativas de prevalência e respectivos intervalos de confiança (IC 95\%) foram calculadas por sexo (masculino, feminino), faixa etária (18 a 24 anos, 25 a 34 anos, 35 a 44 anos, 45 a 54 anos, 55 a 64 anos e 65 ou mais) e nível de escolaridade (0-8, 9-11, 12 ou mais anos de estudo). As análises dos dados foram realizadas com auxílio do aplicativo Stata 11.0 utilizando fatores de ponderação que consideram as diferenças na composição sociodemográfica da amostra do Vigitel em relação à composição da população adulta total de cada cidade, segundo a distribuição censitária do ano 2000. Tais diferenças podem ser decorrentes da cobertura de telefonia fixa desigual nas localidades estudadas, conforme os estratos sociodemográficos.

O inquérito Vigitel foi aprovado pela Comissão Nacional de Ética em Pesquisa em Seres Humanos (Conep). Nas entrevistas telefônicas, o consentimento livre e esclarecido foi substituído pelo consentimento verbal obtido no nomento do contato telefônico com os entrevistados.

\section{Resultados}

Dados de 54.339 adultos foram coletados pelo Vigitel em 2010, sendo 20.764 homens e 33.575 mulheres. A duração média da entrevista foi de 9,5 minutos; a taxa média de sucesso foi de 76,4\% e a taxa de recusa foi de $2,3 \%{ }^{10}$.

A Figura 1 mostra a distribuição de fatores de risco selecionados na população adulta do conjunto das capitais do Brasil e Distrito Federal, segundo sexo (Figura 1a) e escolaridade (Figura $1 b)$.

A prevalência de tabagismo foi de $15,1 \%$ (IC95\% 14,2 - 16,0), maior em homens (17,9\% IC95\% 16,4 - 19,4) do que em mulheres (12,7\% IC95\% 11,7 - 13,7). O mesmo foi verificado para o consumo de carnes com excesso de gordura (homens: 45,5\% IC95\% 43,6 - 47,5; mulheres: 24,5\% IC95\% 23,3 - 25,7), para o consumo de refrigerantes (homens: 30,9\% IC95\% 29,1 - 32,7; mulheres: $25,7 \%$ IC95\% 24,4 - 27,0) e para o consumo abusivo de álcool, cuja prevalência foi mais de duas vezes superior em homens $(26,8 \%$ IC95\% 25,2 - 28,3) do que em mulheres (10,6\% IC95\% 9,7 - 11,4). A prevalência de inatividade fisica foi de $15 \%$, sem diferença entre os sexos (Figura 1a).

A distribuição dos fatores de risco por escolaridade mostrou que a prevalência de fumantes e de consumo de carnes com excesso de gordura foi superior nos adultos com até oito anos de estudo (18,6\% IC95\% 17,1 - 20,2 e 35,7\% IC95\% 33,7 - 37,6, respectivamente) em comparação àqueles que estudaram 12 anos ou mais (10,2\% IC95\% 9,2 - 11,3 e 28,4\% IC95\% 26,8 - 29,9). A prevalência de consumo abusivo de álcool, ao contrário, foi superior entre os mais escolarizados. A inatividade física foi mais frequente entre os adultos com 12 anos ou mais de estudo (15,7\% IC95\% 14,5 - 16,9) em comparação aos indivíduos com 9 a 11 anos de estudo (12,9\% IC95\% $12,0-13,9)$. O consumo de refrigerantes foi mais frequente entre os adultos de escolaridade intermediária (Figura 1b).

Ao comparar as prevalências dos fatores de proteção segundo sexo e escolaridade (Figura 2), a situação esperada seria o inverso do encontrado na distribuição dos fatores de risco. Tal fato se confirmou para o consumo recomendado de frutas e hortaliças e para o uso de proteção contra a radiação solar ultravioleta, mais frequentes em mulheres (Figura 2a) e em adultos de maior escolaridade (Figura 2b). A prevalência de consumo regular de feijão, por sua vez, foi superior em homens e entre os adultos com até oito anos de estudo. Os homens e os adultos de maior escolaridade foram mais fisicamente ativos no tempo livre. 


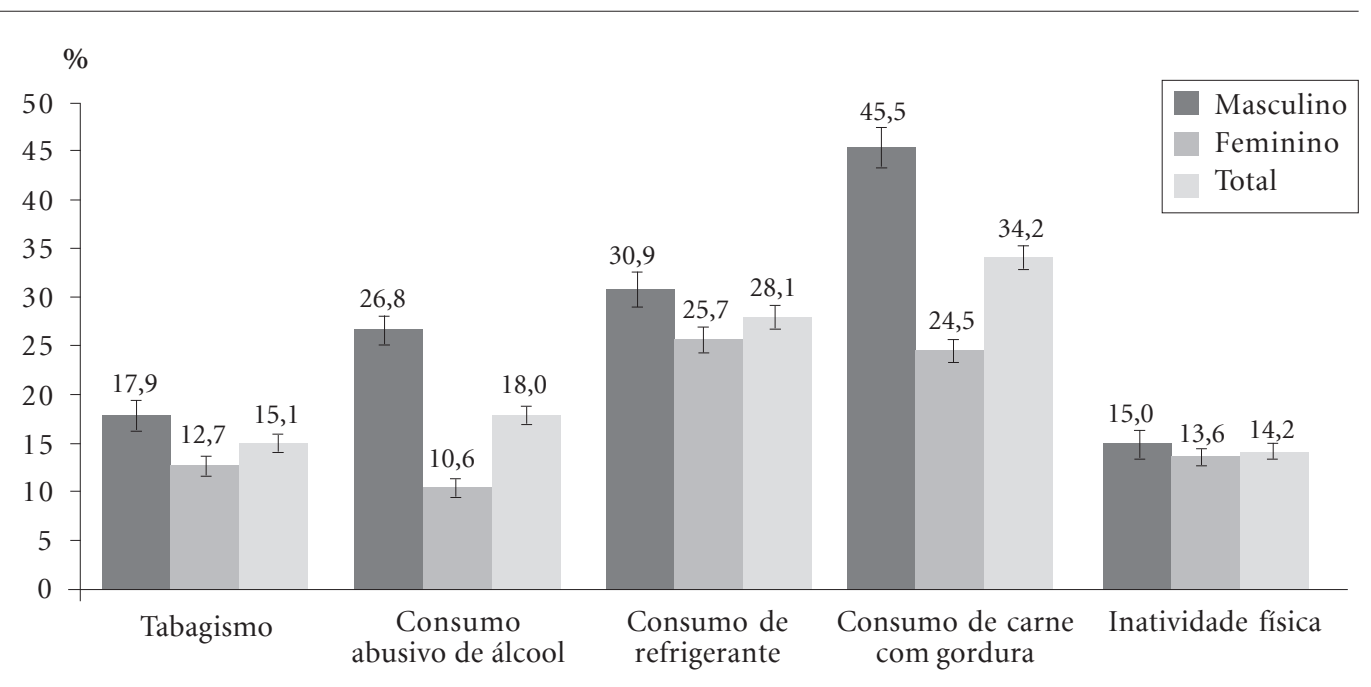

Figura 1a. Prevalência e IC95\% de fatores de risco na população adulta do conjunto das capitais do Brasil, segundo sexo. Vigitel 2010.

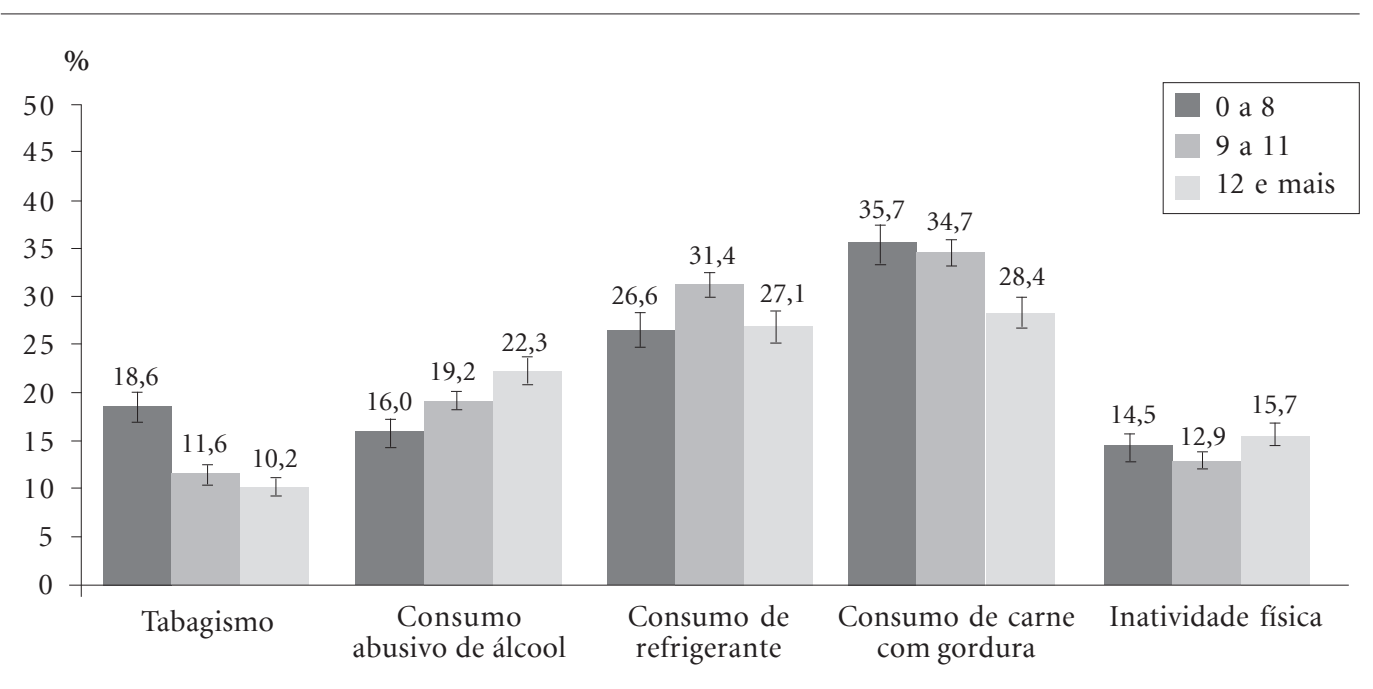

Figura 1b. Prevalência e IC95\% de fatores de risco na população adulta do conjunto das capitais do Brasil, segundo escolaridade. Vigitel 2010.

Fonte: MS/SVS/CGDANT - VIGITEL: Vigilância de Fatores de Risco e Proteção para Doenças Crônicas por Inquérito Telefônico. * Percentuais ponderados para ajustar a distribuição sociodemográfica da amostra Vigitel à distribuição da população adulta de cada cidade no Censo Demográfico de 2000 e para levar em conta o peso populacional de cada cidade. IC $95 \%$ - Intervalo de confiança $(\alpha=0,05)$, levando-se em consideração o efeito do desenho do estudo.

A Tabela 1 apresenta a distribuição dos fatores de risco e proteção para doenças crônicas selecionados segundo sexo e faixa etária. Fatores de risco como consumo de refrigerantes, consumo de carnes com gordura e consumo abusivo de bebidas alcoólicas foram mais frequentes entre os adultos mais jovens, para ambos os sexos.
A menor prevalência de tabagismo foi observada nos indivíduos com 65 anos e mais. Em todas as faixas etárias, as prevalências pontuais desses fatores foram maiores entre os homens. Essas diferenças entre os sexos tenderam a se acentuar com o avanço da idade. Em relação à inatividade física, as prevalências tenderam a aumentar com 
Figura 2a. Prevalência e IC95\% de fatores de proteção na população adulta do conjunto das capitais do Brasil, segundo sexo. Vigitel 2010.

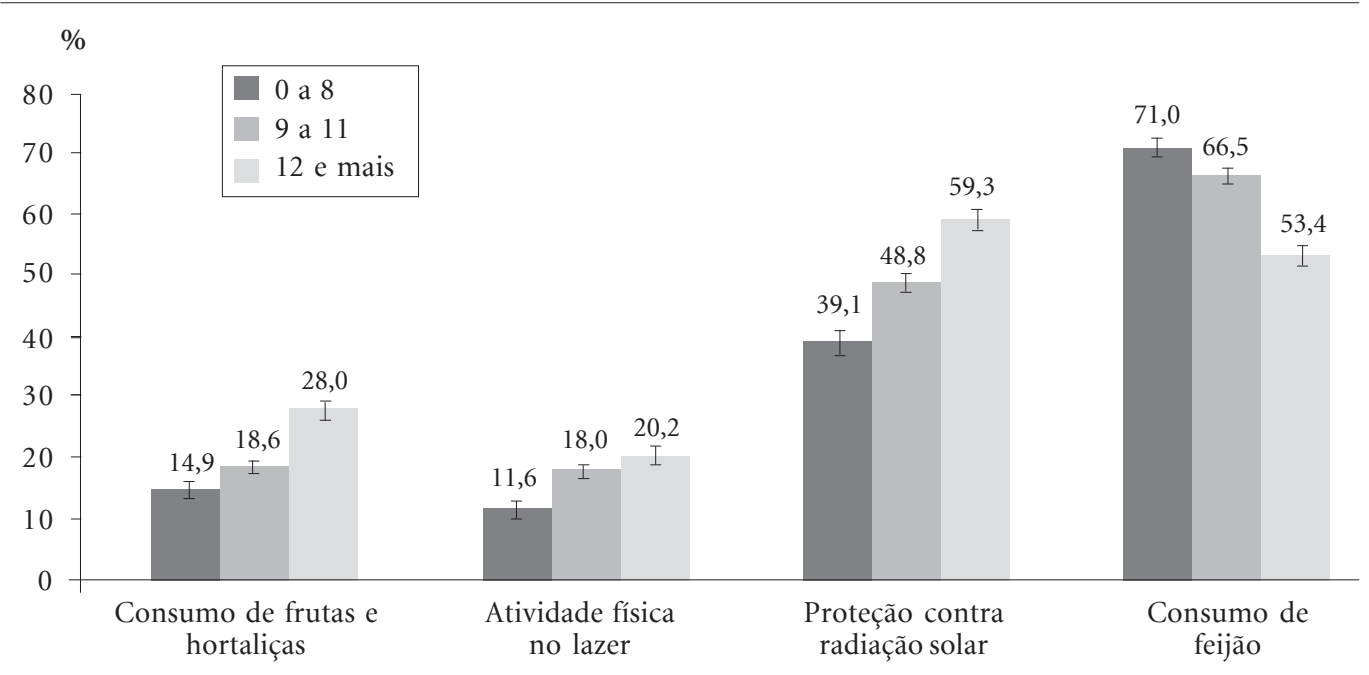

Figura 2b. Prevalência e IC95\% de fatores de proteção na população adulta do conjunto das capitais do Brasil, segundo escolaridade. Vigitel 2010.

Fonte: MS/SVS/CGDANT - VIGITEL: Vigilância de Fatores de Risco e Proteção para Doenças Crônicas por Inquérito Telefônico. *Percentuais ponderados para ajustar a distribuição sociodemográfica da amostra Vigitel à distribuição da população adulta de cada cidade no Censo Demográfico de 2000 e para levar em conta o peso populacional de cada cidade. IC $95 \%$ - Intervalo de confiança $(\alpha=0,05)$, levando-se em consideração o efeito do desenho do estudo.

o passar da idade, sendo nos extremos etários maior nas mulheres e entre os 24 e 64 anos maior nos homens.

A frequência do consumo recomendado de frutas e hortaliças apresentou tendência de aumento com a idade, com maior diferença dos 35-44 anos para os 45-54 anos, quando conside- rados o total e o sexo feminino. Nessas mesmas faixas etárias e para aqueles com 65 anos ou mais, as mulheres apresentaram prevalência maior do que os homens. O consumo de feijão foi mais frequente nos adultos mais jovens, reduzindo entre os adultos de 65 anos ou mais. Em todas as faixas etárias, a prevalência de consumo regular 
Tabela 1. Frequência* (\%) de fatores de risco e proteção para doenças crônicas em adultos, por sexo e faixa etária, no conjunto das capitais do Brasil e no Distrito Federal. Vigitel 2010.

\begin{tabular}{|c|c|c|c|c|c|c|}
\hline & \multicolumn{2}{|c|}{18 a 24 anos } & \multicolumn{2}{|c|}{25 a 34 anos } & \multicolumn{2}{|c|}{35 a 44 anos } \\
\hline & $\%$ & IC 95\% & $\%$ & IC 95\% & $\%$ & IC 95\% \\
\hline \multicolumn{7}{|l|}{ Tabagismo } \\
\hline Total & 12,5 & $10,5-14,6$ & 17,3 & $15,0-19,6$ & 15,2 & $13,7-16,8$ \\
\hline Homens & 12,7 & $10,0-15,3$ & 23,4 & $19,4-27,4$ & 16,6 & $14,2-19,1$ \\
\hline Mulheres & 12,4 & $9,4-15,5$ & 11,8 & $9,6-14,0$ & 14,0 & $12,0-16,1$ \\
\hline \multicolumn{7}{|c|}{ Consumo de frutas e hortaliças recomendado } \\
\hline Total & 14,5 & $12,2-16,8$ & 16,2 & $14,3-18,1$ & 17,9 & $16,4-19,4$ \\
\hline Homens & 12,9 & $9,0-16,8$ & 14,4 & $11,1-17,7$ & 15,1 & $13,0-17,2$ \\
\hline Mulheres & 16,0 & $13,4-18,7$ & 17,7 & $15,7-19,8$ & 20,4 & $18,3-22,4$ \\
\hline \multicolumn{7}{|c|}{ Consumo de feijão } \\
\hline Total & 68,2 & $65,1-71,2$ & 67,9 & $65,8-70,1$ & 67,4 & $65,6-69,2$ \\
\hline Homens & 75,6 & $71,6-79,6$ & 72,0 & $68,7-75,4$ & 71,1 & $68,4-73,9$ \\
\hline Mulheres & 61,3 & $57,1-65,5$ & 64,2 & $61,5-66,9$ & 64,1 & $61,8-66,5$ \\
\hline \multicolumn{7}{|c|}{ Consumo de refrigerantes } \\
\hline Total & 36,7 & $33,4-40,1$ & 34,1 & $31,8-36,4$ & 29,2 & $27,3-31,1$ \\
\hline Homens & 37,0 & $31,7-42,4$ & 37,5 & $33,8-41,3$ & 32,8 & $29,8-35,9$ \\
\hline Mulheres & 36,5 & $32,2-40,7$ & 31,0 & $28,3-33,8$ & 26,0 & $23,7-28,4$ \\
\hline \multicolumn{7}{|c|}{ Consumo de carnes com excesso de gordura } \\
\hline Total & 39,6 & $36,1-43,1$ & 39,5 & $37,1-42,0$ & 35,0 & $33,1-37,0$ \\
\hline Homens & 52,2 & $46,4-58,0$ & 51,0 & $47,1-55,0$ & 44,6 & $41,5-47,7$ \\
\hline Mulheres & 28,0 & $24,4-31,5$ & 29,1 & $26,4-31,9$ & 26,7 & $24,4-29,0$ \\
\hline \multicolumn{7}{|c|}{ Atividade física no lazer } \\
\hline Total & 20,4 & $17,6-23,2$ & 14,8 & $13,2-16,4$ & 12,6 & $11,3-13,9$ \\
\hline Homens & 29,5 & $24,5-34,5$ & 17,8 & $15,0-20,6$ & 14,1 & $11,9-16,3$ \\
\hline Mulheres & 12,0 & $9,4-14,6$ & 12,1 & $10,4-13,8$ & 11,3 & $9,7-12,9$ \\
\hline \multicolumn{7}{|c|}{ Inatividade física } \\
\hline Total & 12,0 & $9,3-14,7$ & 9,7 & $8,4-11,0$ & 10,9 & $9,8-12,1$ \\
\hline Homens & 9,1 & $4,2-14,0$ & 11,0 & $8,7-13,3$ & 13,3 & $11,3-15,3$ \\
\hline Mulheres & 14,6 & $12,0-17,3$ & 8,5 & $7,3-9,8$ & 8,8 & $7,5-10,2$ \\
\hline \multicolumn{7}{|c|}{ Consumo abusivo de bebidas alcoólicas } \\
\hline Total & 20,3 & $17,7-22,8$ & 23,2 & $21,3-25,2$ & 19,5 & $18,0-21,1$ \\
\hline Homens & 26,3 & $21,8-30,9$ & 34,0 & $30,5-37,4$ & 30,0 & $27,2-32,8$ \\
\hline Mulheres & 14,6 & $11,9-17,3$ & 13,5 & $11,6-15,4$ & 10,4 & $8,9-11,8$ \\
\hline
\end{tabular}

de feijão foi maior entre os homens. A prevalência de atividade física no lazer foi maior entre os mais jovens (18 a 24 anos), quando considerados ambos os sexos e nos homens, sendo em todas as faixas etárias menor entre as mulheres (Tabela 1).

Na Tabela 2 são apresentadas as frequências de excesso de peso e obesidade, autoavaliação de saúde ruim, morbidades referidas e uso de proteção contra radiação solar, de acordo com sexo e faixa etária. $\mathrm{O}$ excesso de peso e a obesidade aumentaram com a idade até os 45-54 anos. Para o excesso de peso, as diferenças entre os sexos foram mais acentuadas nos mais jovens, com maiores prevalências entre os homens; para a obesidade, diferenças entre os sexos ocorreram a partir dos 55 anos de idade, com maiores prevalências entre as mulheres.

A autoavaliação de saúde ruim aumentou entre as mulheres aos 45 anos, idade a partir da qual a frequência em mulheres passa a ser maior do que a frequencia em homens (Tabela 2). O uso de proteção contra a radiação solar foi menor nos indivíduos de 18 a 24 anos, sem diferenças significativas após os 34 anos de idade. Em todas as faixas etárias, a frequência do uso de proteção solar foi superior nas mulheres, chegando a ser $50 \%$ maior entre os 18 e 54 anos.

Para ambos os sexos, as prevalências de hipertensão arterial e diabetes aumentaram com o 
Tabela 1. continuação

\begin{tabular}{|c|c|c|c|c|c|c|}
\hline & \multicolumn{2}{|c|}{45 a 54 anos } & \multicolumn{2}{|c|}{55 a 64 anos } & \multicolumn{2}{|c|}{$>65$ anos } \\
\hline & $\%$ & IC $95 \%$ & $\%$ & IC $95 \%$ & $\%$ & IC $95 \%$ \\
\hline \multicolumn{7}{|l|}{ Tabagismo } \\
\hline Total & 18,3 & $16,4-20,2$ & 16,5 & $14,3-18,7$ & 8,1 & $6,7-9,5$ \\
\hline Homens & 20,5 & $17,4-23,6$ & 20,3 & $16,3-24,4$ & 10,6 & $7,6-13,5$ \\
\hline Mulheres & 16,4 & $14,1-18,8$ & 13,4 & $11,2-15,6$ & 6,5 & $5,2-7,9$ \\
\hline \multicolumn{7}{|c|}{ Consumo de frutas e hortaliças recomendado } \\
\hline Total & 21,5 & $19,7-23,2$ & 24,6 & $22,2-26,9$ & 21,8 & $19,9-23,7$ \\
\hline Homens & 16,9 & $14,3-19,4$ & 22,2 & $18,1-26,2$ & 17,2 & $14,3-20,2$ \\
\hline Mulheres & 25,3 & $22,9-27,7$ & 26,4 & $23,7-29,2$ & 24,7 & $22,2-27,1$ \\
\hline \multicolumn{7}{|c|}{ Consumo de feijão } \\
\hline Total & 65,2 & $63,2-67,2$ & 66,0 & $63,6-68,3$ & 61,1 & $58,8-63,3$ \\
\hline Homens & 70,9 & $67,9-74,0$ & 71,9 & $68,3-75,4$ & 68,1 & $64,3-71,9$ \\
\hline Mulheres & 60,4 & $57,7-63,1$ & 61,3 & $58,3-64,3$ & 56,6 & $53,9-59,3$ \\
\hline \multicolumn{7}{|c|}{ Consumo de refrigerantes } \\
\hline Total & 20,5 & $18,6-22,3$ & 14,4 & $12,6-16,2$ & 13,0 & $11,3-14,6$ \\
\hline Homens & 22,3 & $19,3-25,2$ & 15,9 & $13,0-18,8$ & 16,7 & $13,5-19,9$ \\
\hline Mulheres & 19,0 & $16,7-21,3$ & 13,2 & $10,9-15,5$ & 10,6 & $8,9-12,3$ \\
\hline \multicolumn{7}{|c|}{ Consumo de carnes com excesso de gordura } \\
\hline Total & 30,8 & $28,6-32,9$ & 23,9 & $21,5-26,3$ & 18,9 & $17,1-20,8$ \\
\hline Homens & 41,1 & $37,6-44,6$ & 35,6 & $31,3-39,8$ & 28,2 & $24,5-31,9$ \\
\hline Mulheres & 22,1 & $19,6-24,6$ & 14,6 & $12,2-17,1$ & 13,1 & $11,2-15,0$ \\
\hline \multicolumn{7}{|c|}{ Atividade física no lazer } \\
\hline Total & 13,1 & $11,6-14,5$ & 13,1 & $11,6-14,6$ & 12,2 & $10,7-13,8$ \\
\hline Homens & 13,3 & $10,9-15,7$ & 14,7 & $12,2-17,2$ & 16,6 & $13,7-19,6$ \\
\hline Mulheres & 12,9 & $11,2-14,6$ & 11,8 & $9,9-13,8$ & 9,4 & $7,7-11,1$ \\
\hline \multicolumn{7}{|c|}{ Inatividade física } \\
\hline Total & 13,5 & $12,0-15,1$ & 20,0 & $17,8-22,1$ & 36,7 & $34,4-39,0$ \\
\hline Homens & 17,9 & $15,0-20,7$ & 24,5 & $20,5-28,5$ & 35,8 & $31,8-39,8$ \\
\hline Mulheres & 9,8 & $8,4-11,3$ & 16,4 & $14,3-18,5$ & 37,3 & $34,5-40,0$ \\
\hline \multicolumn{7}{|c|}{ Consumo abusivo de bebidas alcoólicas } \\
\hline Total & 16,1 & $14,6-17,6$ & 10,4 & $8,9-11,9$ & 4,5 & $3,5-5,5$ \\
\hline Homens & 23,6 & $21,0-26,2$ & 17,8 & $14,6-20,9$ & 9,0 & $6,7-11,3$ \\
\hline Mulheres & 9,8 & $8,2-11,4$ & 4,6 & $3,6-5,6$ & 1,8 & $1,1-2,4$ \\
\hline
\end{tabular}

Fonte: MS/SVS/CGDANT - VIGITEL: Vigilância de Fatores de Risco e Proteção para Doenças Crônicas por Inquérito Telefônico. Percentuais ponderados para ajustar a distribuição sociodemográfica da amostra Vigitel à distribuição da população adulta de cada cidade no Censo Demográfico de 2000 e para levar em conta o peso populacional de cada cidade. IC95\% - Intervalo de confiança $(\alpha=$ 0,05), levando-se em consideração o efeito do desenho do estudo.

avanço da idade, com maior aumento a partir dos 35 anos de idade para hipertensão e a partir dos 45 anos para diabetes. A partir dos 55 anos, a prevalência de hipertensão foi maior entre as mulheres. Para o diabetes, não foram verificadas diferenças expressivas entre os sexos, embora as estimativas pontuais tenham sido maiores para as mulheres. Asma, bronquite asmática, bronquite crônica e enfisema apresentaram comportamento inverso, com tendência de menores estimativas pontuais nas maiores faixas etárias. Entre os 25 e 44 anos, a prevalência dessas doenças entre as mulheres foi maior do que nos homens.

\section{Discussão}

Os principais resultados desse estudo apontam para diferenças na distribuição de fatores de risco e proteção para doenças crônicas de acordo com as características sociodemográficas estudadas - sexo, faixa etária e escolaridade. Em geral, fatores de risco como tabagismo, consumo de refrigerantes e de carnes com gordura foram mais prevalentes em homens, adultos de menor escolaridade e mais jovens, enquanto fatores de proteção como consumo de frutas e hortaliças e uso de proteção solar foram mais frequentes em mulheres, indivíduos mais escolarizados e mais 
Tabela 2. Frequência* ${ }^{*} \%$ de excesso de peso e obesidade, autoavaliação de saúde, morbidade referida e uso de proteção solar em adultos, por sexo e faixa etária, no conjunto das capitais do Brasil e no Distrito Federal. Vigitel 2010.

\begin{tabular}{|c|c|c|c|c|c|c|}
\hline & \multicolumn{2}{|c|}{18 a 24 anos } & \multicolumn{2}{|c|}{25 a 34 anos } & \multicolumn{2}{|c|}{35 a 44 anos } \\
\hline & $\%$ & IC $95 \%$ & $\%$ & IC 95\% & $\%$ & IC $95 \%$ \\
\hline \multicolumn{7}{|c|}{ Excesso de peso } \\
\hline Total & 29,6 & $25,6-33,6$ & 44,5 & $42,0-47,0$ & 52,7 & $50,6-54,8$ \\
\hline Homens & 31,6 & $25,2-38,0$ & 50,3 & $46,3-54,3$ & 58,5 & $55,4-61,7$ \\
\hline Mulheres & 27,6 & $22,8-32,5$ & 38,6 & $35,7-41,6$ & 46,9 & $44,2-49,7$ \\
\hline \multicolumn{7}{|l|}{ Obesidade } \\
\hline Total & 6,3 & $4,3-8,2$ & 13,3 & $11,7-14,9$ & 16,6 & $15,1-18,1$ \\
\hline Homens & 5,6 & $2,6-8,5$ & 13,5 & $11,0-16,0$ & 16,8 & $14,6-19,0$ \\
\hline Mulheres & 7,0 & $4,4-9,6$ & 13,2 & $11,2-15,1$ & 16,3 & $14,3-18,3$ \\
\hline \multicolumn{7}{|c|}{ Autoavaliaçao de saúde ruim } \\
\hline Total & 3,7 & $2,3-5,0$ & 3,2 & $2,4-4,0$ & 3,9 & $3,0-4,7$ \\
\hline Homens & 3,5 & $1,2-5,8$ & 2,3 & $1,2-3,3$ & 3,0 & $1,7-4,4$ \\
\hline Mulheres & 3,9 & $2,4-5,3$ & 4,0 & $2,8-5,3$ & 4,6 & $3,6-5,6$ \\
\hline \multicolumn{7}{|c|}{ Hipertensão arterial } \\
\hline Total & 8,2 & $5,3-11,1$ & 10,0 & $8,6-11,4$ & 18,7 & $17,1-20,3$ \\
\hline Homens & 7,2 & $2,2-12,2$ & 9,4 & $7,2-11,6$ & 17,9 & $15,5-20,2$ \\
\hline Mulheres & 9,1 & $6,1-12,2$ & 10,5 & $8,6-12,4$ & 19,5 & $17,3-21,7$ \\
\hline \multicolumn{7}{|l|}{ Diabetes } \\
\hline Total & 1,7 & $0,6-2,8$ & 2,4 & $1,6-3,1$ & 3,5 & $2,8-4,2$ \\
\hline Homens & 0,7 & $0,2-1,3$ & 1,7 & $0,9-2,6$ & 3,3 & $2,3-4,4$ \\
\hline Mulheres & 2,6 & $0,6-4,7$ & 3,0 & $1,8-4,1$ & 3,7 & $1,8-4,1$ \\
\hline \multicolumn{7}{|l|}{ Asma } \\
\hline Total & 9,2 & $7,2-11,1$ & 6,2 & $5,4-7,0$ & 5,5 & $4,6-6,4$ \\
\hline Homens & 10,2 & $6,6-13,8$ & 4,6 & $3,7-5,6$ & 3,8 & $2,7-5,0$ \\
\hline Mulheres & 8,2 & $6,6-9,9$ & 7,6 & $6,3-8,9$ & 7,0 & $5,6-8,4$ \\
\hline \multicolumn{7}{|c|}{ Proteção contra radiação solar } \\
\hline Total & 39,0 & $35,6-42,5$ & 45,2 & $42,8-47,7$ & 49,2 & $47,2-51,2$ \\
\hline Homens & 29,8 & $24,8-34,8$ & 35,5 & $31,9-39,2$ & 39,8 & $36,8-42,9$ \\
\hline Mulheres & 47,6 & $43,2-52,0$ & 54,0 & $50,9-57,1$ & 57,4 & $54,8-59,9$ \\
\hline
\end{tabular}

velhos. As diferenças entre os sexos se acentuaram com o avanço da idade. A prevalência de excesso de peso e de obesidade, a inatividade física e a autoavaliação ruim do estado de saúde aumentaram com a idade, mesma situação verificada para o relato de morbidades como hipertensão arterial e diabetes. O consumo abusivo de álcool, a atividade física no lazer e o consumo regular de feijão foram mais frequentes entre os mais jovens.

$\mathrm{O}$ acompanhamento das tendências dos fatores de risco e proteção para doenças crônicas ao longo do tempo se faz importante para o planejamento e posterior avaliação de políticas públicas voltadas à promoção de saúde da população. Dados semelhantes aos aqui apresentados já haviam sido destacados em publicações com dados do inquérito telefônico nacional e de ou- tros inquéritos de saúde em anos anteriores, mostrando a consistência dos resultados do Vigitel ao longo dos anos ${ }^{11-15}$ e em relação a diferentes inquéritos nacionais, apesar das diferenças metodológicas ${ }^{16,17}$.

A prevalência de tabagismo verificada em 2010 corrobora a tendência de queda no número de fumantes em todo o país ${ }^{15,18}$. Os homens ainda são os que mais fumam, mas as tendências indicam que são eles que apresentam uma redução mais expressiva deste hábito ${ }^{11}$.

Em sentido inverso, as altas prevalências de excesso de peso e obesidade estão entre os dados mais preocupantes. Os valores estimados pelo Vigitel em 2010, decorrentes do autorrelato de peso e altura, são semelhantes aos de inquéritos domiciliares que realizaram as medições para cálculo do $\mathrm{IMC}^{19}$ e da mesma forma confirmam 
Tabela 2. continuação

\begin{tabular}{|c|c|c|c|c|c|c|}
\hline & \multicolumn{2}{|c|}{45 a 54 anos } & \multicolumn{2}{|c|}{55 a 64 anos } & \multicolumn{2}{|c|}{$>65$ anos } \\
\hline & $\%$ & IC 95\% & $\%$ & IC 95\% & $\%$ & IC 95\% \\
\hline \multicolumn{7}{|c|}{ Excesso de peso } \\
\hline Total & 59,3 & $57,0-61,5$ & 61,7 & $59,0-64,3$ & 58,4 & $55,9-60,9$ \\
\hline Homens & 64,2 & $60,8-67,6$ & 63,0 & $58,9-67,2$ & 58,8 & $54,6-62,9$ \\
\hline Mulheres & 54,9 & $51,6-57,6$ & 60,4 & $57,0-63,8$ & 58,1 & $54,9-61,2$ \\
\hline \multicolumn{7}{|l|}{ Obesidade } \\
\hline Total & 21,6 & $19,6-23,5$ & 20,6 & $18,4-22,7$ & 19,4 & $17,2-21,7$ \\
\hline Homens & 22,5 & $19,4-25,6$ & 16,6 & $13,9-19,3$ & 16,3 & $12,8-19,9$ \\
\hline Mulheres & 20,7 & $18,2-23,2$ & 24,1 & $20,9-27,3$ & 21,8 & $19,0-24,7$ \\
\hline \multicolumn{7}{|c|}{ Autoavaliaçao de saúde ruim } \\
\hline Total & 6,5 & $5,3-7,8$ & 8,1 & $6,7-9,5$ & 7,2 & $6,2-8,2$ \\
\hline Homens & 5,2 & $3,2-7,1$ & 5,0 & $3,5-6,5$ & 3,8 & $2,8-4,7$ \\
\hline Mulheres & 7,7 & $6,1-9,2$ & 10,5 & $8,3-12,7$ & 9,3 & $7,8-10,8$ \\
\hline \multicolumn{7}{|c|}{ Hipertensão arterial } \\
\hline Total & 35,8 & $33,7-38,0$ & 52,8 & $50,2-55,4$ & 60,2 & $57,8-62,5$ \\
\hline Homens & 34,2 & $30,8-37,6$ & 48,1 & $43,8-52,5$ & 53,0 & $48,8-57,2$ \\
\hline Mulheres & 37,2 & $34,5-40,0$ & 56,5 & $53,4-59,6$ & 64,7 & $62,0-67,3$ \\
\hline \multicolumn{7}{|l|}{ Diabetes } \\
\hline Total & 8,3 & $7,1-9,6$ & 16,4 & $14,5-18,3$ & 22,0 & $20,0-24,0$ \\
\hline Homens & 7,7 & $5,9-9,4$ & 18,2 & $14,9-21,5$ & 20,2 & $16,9-23,5$ \\
\hline Mulheres & 8,9 & $7,2-10,6$ & 15,0 & $12,9-17,1$ & 23,2 & $20,7-25,6$ \\
\hline \multicolumn{7}{|l|}{ Asma } \\
\hline Total & 6,4 & $5,3-7,6$ & 7,3 & $5,7-9,0$ & 6,4 & $5,4-7,4$ \\
\hline Homens & 5,3 & $3,4-7,1$ & 6,2 & $3,2-9,3$ & 5,2 & $3,6-6,9$ \\
\hline Mulheres & 7,4 & $6,1-8,8$ & 8,2 & $6,4-10,0$ & 7,1 & $5,9-8,4$ \\
\hline \multicolumn{7}{|c|}{ Proteção contra radiação solar } \\
\hline Total & 47,4 & $45,2-49,6$ & 47,3 & $44,7-49,9$ & 46,5 & $44,1-48,8$ \\
\hline Homens & 36,1 & $32,9-39,3$ & 40,1 & $35,9-44,2$ & 39,0 & $35,1-43,0$ \\
\hline Mulheres & 56,9 & $54,0-59,7$ & 53,1 & $49,8-56,3$ & 51,2 & $48,4-54,0$ \\
\hline
\end{tabular}

Fonte: MS/SVS/CGDANT - VIGITEL: Vigilância de Fatores de Risco e Proteção para Doenças Crônicas por Inquérito Telefônico. * Percentuais ponderados para ajustar a distribuição sociodemográfica da amostra Vigitel à distribuição da população adulta de cada cidade no Censo Demográfico de 2000 e para levar em conta o peso populacional de cada cidade. IC $95 \%$ - Intervalo de confiança $(\alpha=0,05)$, levando-se em consideração o efeito do desenho do estudo.

as tendências crescentes de excesso de peso e obesidade verificadas globalmente ${ }^{2,4}$.

As consequências do excesso de peso na saúde da população são amplamente conhecidas ${ }^{3,20}$, bem como seus determinantes ${ }^{2,21}$. Os dados do VIGITEL mostram estilos de vida insalubres nas capitais do país, como o baixo consumo de alimentos saudáveis, baixa proporção de ativos no lazer e elevada proporção de inativos. O consumo de frutas e hortaliças no Brasil não chega à metade do recomendado pela Organização Mundial da Saúde ${ }^{21,22}$. Estudos recentes indicam que a frequência de consumo desses alimentos não tem alterado nos últimos anos ${ }^{23,24}$, apesar da existência de políticas de incentivo à alimentação saudável, reforçando que novas estratégias precisam ser acionadas para incentivar esse hábito ${ }^{6}$. A re- dução do preço desses alimentos, por exemplo, pode ser considerada uma medida de impacto para o aumento do seu consumo na população brasileira ${ }^{25}$. A Pesquisa de Orçamentos Familiares (2008-2009) também mostrou que mulheres e indivíduos mais velhos e de maior escolaridade apresentaram maior consumo de cinco porções diárias de frutas e hortaliças, o que pode estar associado ao maior acesso à informação e à maior preocupação com a saúde desse estrato da população ${ }^{22,26}$. Ainda para os idosos, a maior prevalência de consumo de frutas e hortaliças pode ser influenciada por hábitos mais saudáveis adquiridos no passado, anteriores ao fenômeno atual de comidas rápidas, industrializadas, e também por seguirem orientações recebidas dos profissionais de saúde, em função da sobrecarga 
de fatores de risco e da presença de comorbida$\operatorname{des}^{27}$. Esses mesmos fatores podem explicar a redução do consumo de carnes com excesso de gordura e de refrigerantes com o aumento da idade e da escolaridade.

Diferentemente do observado para outros itens da dieta, o feijão, marcador de uma alimentação saudável pelo alto teor de fibras e de ferro, é mais consumido entre os homens e os adultos mais jovens. Apesar de ainda apresentar prevalência alta de consumo, provavelmente por ser um alimento típico da dieta brasileira, estudos recentes indicam uma redução do seu consumo nos últimos $\operatorname{anos}^{28}$, em concordância com os dados da Pesquisa de Orçamentos Familiares que indica a diminuição da disponibilidade desse alimento nos lares brasileiros ${ }^{22}$.

A maior frequência de atividade física no lazer entre os homens e mais escolarizados, já verificada em estudos anteriores ${ }^{29,30}$ contrasta com as maiores frequências de inatividade física também entre os mais escolarizados, sem diferenças expressivas entre os sexos. Essa aparente contradição pode ser explicada pela composição de cada indicador, em que a inatividade física considera quatro diferentes domínios, sendo a atividade do tempo livre ou lazer apenas um deles. Assim, a participação maior das mulheres nas atividades domésticas deve suavizar as diferenças entre os sexos no indicador de inatividade ${ }^{29}$. As diferenças desses dois indicadores com relação à idade também estão relacionadas a esse fato, considerando que os indivíduos mais velhos, que não mais trabalham e/ou estudam, podem apresentar "desvantagens" no indicador composto ${ }^{31}$.

O consumo abusivo de álcool é fator de risco para diversas doenças como hipertensão arterial, cirrose, acidente vascular cerebral, câncer de faringe, laringe, esôfago e fígado, além das causas externas ${ }^{2,32}$. O consumo de álcool no país é elevado, com tendência crescente ${ }^{33}$, justificando a intensificação de medidas legislativas e fiscalizatórias $^{6}$. O Brasil apresenta maior prevalência de consumo abusivo de álcool em adultos em comparação aos Estados Unidos ${ }^{34}$ e Argentina mas ainda menor do que o Chile ${ }^{35}$. O consumo maior em homens do que em mulheres é um achado recorrente ${ }^{33,34,36}$. O maior consumo de bebidas alcoólicas entre os mais escolarizados já foi demonstrado como fator independente da ida$\mathrm{de}^{37,38}$, embora relação inversa também seja encontrada na literatura ${ }^{36,39}$.

O aumento da prevalência de doenças crônicas como hipertensão arterial e diabetes com a idade é compatível com o aumento do risco para
DCNT em populações idosas pelo acúmulo de fatores de risco ${ }^{2,40,41}$. As maiores frequências dessas doenças nas mulheres pode estar relacionada à maior procura aos serviços e maior oportunidade de diagnósticos médicos ${ }^{42-44}$ nessa população, tendo em vista tratar-se de informação autorreferida. Não há consenso na literatura sobre diferenças na ocorrência dessas doenças entre os $\operatorname{sexos}^{16,45-47}$, mas no Brasil verifica-se uma tendência de maior ocorrência de ambas nas mulheres $^{48-50}$.

Diferentemente das outras doenças crônicas, a prevalência de asma/bronquite /enfisema tendeu a ser maior entre os adultos mais jovens, semelhante à relação verificada nos resultados do inquérito telefônico dos Estados Unidos ${ }^{34}$. Na Pesquisa Mundial de Saúde em $2003^{41}$ não foram verificadas diferenças significativas na prevalência de asma com a idade. A tendência de maior ocorrência da doença entre as mulheres também foi verificada em outros estudos brasileiros ${ }^{51,52}$. A ocorrência de asma tem sido relacionada à industrialização e à adoção de um estilo de vida moder$\mathrm{no}^{53}$. Avaliação feita com os resultados da Pesquisa Nacional por Amostra de Domicílios (PNAD) mostrou uma tendência de diminuição na prevalência de asma nos últimos anos entre idosos (> 60 anos) $)^{52}$, em contraposição ao aumento em crianças e adolescentes, no mesmo período ${ }^{54}$.

A autoavaliação de saúde ruim ou muito ruim é um indicador de necessidades em saúde da população. A literatura aponta pior avaliação de saúde entre mulheres, idosos e indivíduos de menor escolaridade, baixa renda e portadores de doenças crônicas ${ }^{5-57}$. Os resultados aqui apresentados são coerentes com o conhecimento atual, tendo em vista que os fatores avaliados estão correlacionados. As mulheres, os indivíduos de maior idade e portadores de doenças são também os que mais acessam os serviços e as informações de saúde, tendo assim maior preocupação com a mesma. Os homens, ao contrário, tendem a ter uma avaliação de saúde ruim na presença de doenças crônicas ${ }^{48}$.

A proteção à exposição solar não é um hábito comum no Brasil, e deve ser incentivado tendo em vista a alta ocorrência de câncer de pele não melanoma no país, representando $25 \%$ de todos os tumores malignos registrados ${ }^{58}$. Menos da metade da população adulta brasileira referiu se proteger contra a radiação ultravioleta quando exposta ao sol por mais de 30 minutos. Dados semelhantes foram encontrados em inquérito domiciliar em Anchieta (ES), cidade litorânea do Sudeste do país ${ }^{51}$. As mulheres e adul- 
tos com 12 anos e mais de estudo se protegem mais contra os raios solares, refletindo maior cuidado em saúde ou mesmo preocupação estética dessa parcela da população. O maior percentual de proteção solar nas pessoas de maior escolaridade também pode ser devido ao custo, quando se refere ao uso do filtro solar ${ }^{59,60}$. Além disso, a exposição solar de homens e pessoas de menor escolaridade é, em geral, associada a atividades de trabalho, enquanto a maior exposição solar de indivíduos com 12 anos ou mais de estudo pode ser relacionada a atividades de lazer, embora sejam verificadas diferenças regionais ${ }^{59,60}$. Mesmo em Florianópolis, cidade litorânea no sul do país que apresenta um dos maiores percentuais de proteção solar, essa associação é encontrada: os homens e jovens são os que mais se expõe ao sol, e menos se protegem ${ }^{60}$. No Vigitel em 2010, não foram encontradas diferenças marcantes em relação à idade da população, talvez por se restringir a indivíduos adultos, enquanto a exposição solar de jovens tem sido associada a atividades esportivas e escolares ${ }^{59,61}$.

Dentre as limitações do estudo destaca-se o fato da amostra se restringir àqueles que possuem telefone fixo, com menores coberturas nas regiões Norte e Nordeste. No entanto, o uso de pesos de pós-estratificação reduz o viés, buscando aproximar a amostra do estudo da população das capitais do Brasil, segundo o censo demográfico de 2000. Estima-se que tenham ocorrido recentes mudanças na distribuição da escolaridade e faixas etárias no país, com incremento na população de idosos, entretanto, ainda foram adotados os pesos do censo do ano 2000. Torna-se necessária a atualização futura das estimativas do Vigitel com os dados do censo de 2010. Outra limitação refere-se ao uso da morbidade autorreferida, dependente do acesso ao diagnóstico médico e do entendimento do indivíduo sobre sua condição de saúde. Estudos que tem avaliado essas informações, no entanto, indicam que essas estimativas podem ser utilizadas $^{49,62}$, com a vantagem da facilidade de obtenção e do menor custo ${ }^{63}$.

A vigilância de fatores de risco de DCNT pelo VIGITEL tem se constituído uma ferramenta epidemiológica relevante para apoiar políticas de promoção à saúde e prevenção das DCNT. O aumento da prevalência de um grande número de fatores de risco e de morbidade com o avanço da idade indica a necessidade de atuação em todos os ciclos de vida, de modo a diminuir a carga das doenças crônicas e suas consequências na qualidade de vida da população. Dessa forma, o Plano de Ações Estratégicas para o Enfrentamento das Doenças Crônicas não transmissíveis no Brasil estabelece ações de promoção de saúde e cuidado integral desde a gestação até a população idosa ${ }^{6}$.

\section{Conclusão}

Os resultados do inquérito telefônico Vigitel em 2010 apontam diferentes comportamentos em saúde da população segundo características sociodemográficas. Fatores de risco tendem a ser mais frequentes em homens e individuos de menor escolaridade e, em sua maioria, entre adultos mais jovens. Entre os fatores de proteção, o consumo de frutas e hortaliças costuma aumentar com a idade, enquanto reduz a frequencia de atividade fisica. Essa distribuição desigual dos fatores de risco e proteção para doenças crônicas na população e suas particularidades deve ser considerada nas estratégias de enfrentamento dessas doenças, o que tem sido feito a partir das ações e programas prioritários estabelecidos no Plano de Ações Estratégicas para o Enfrentamento das Doenças Crônicas não transmissíveis no Brasil (2011-2022).

\section{Colaboradores}

Todos os autores participaram da concepção do estudo. BPM Iser revisou a literatura, participou das análises e interpretação dos dados e redigiu o manuscrito. RT de C Yokota, NNB de Sá participaram das análises e interpretação dos dados e redação do manuscrito. L de Moura e DC Malta participaram da interpretação dos dados e redação do manuscrito. 


\section{Referências}

1. Beaglehole R, Ebrahim S, Reddy S, Voute J, Leeder S. Prevention of chronic diseases: a call to action. Lancet 2007; 370(9605):2152-2157.

2. World Health Organization (WHO). Global status report on noncommunicable diseases 2010. Geneva: WHO; 2011.

3. Alwan A, Maclean DR, Riley LM, d'Espaignet ET, Mathers CD, Stevens GA, Bettcher D. Monitoring and surveillance of chronic non-communicable diseases: progress and capacity in high-burden countries. Lancet 2010; 376(9755):1861-1868.

4. Schmidt MI, Duncan BB, Azevedo e Silva G, Menezes AM, Monteiro CA, Barreto SM, Chor D, Menezes PR. Chronic non-communicable diseases in Brazil: burden and current challenges. Lancet 2011; 377(9781):1949-1961.

5. Brasil. Ministério da Saúde. Sistema de Informações Hospitalares do SUS (SIH/SUS). Brasília: Datasus; 2010.

6. Brasil. Ministério da Saúde (MS). Secretaria de Vigilância em Saúde. Departamento de Análise de Situação de Saúde. Plano de ações estratégicas para o enfrentamento das doenças crônicas não transmissíveis (DCNT) no Brasil 2011-2022. Brasília: MS; 2011.

7. World Health Organization (WHO). Global health risks: mortality and burden of disease attributable to selected major risks. Geneva: WHO; 2009.

8. Moura EC, Neto OL, Malta DC, Moura L, Silva NN, Bernal R, Claro RF, Monteiro CA. Vigilância de Fatores de Risco para Doenças Crônicas por Inquérito Telefônico nas capitais dos 26 estados brasileiros e no Distrito Federal (2006). Rev Bras Epidemiol 2008; 11(Supl. 1):20-37.

9. Brasil. Ministério da Saúde (MS). Departamento de Análise de Situação de Saúde. Vigilância de Fatores de Risco e Proteção para Doenças Crônicas por Inquérito Telefônico, Vigitel 2007. Brasília, DF: MS; 2008.

10. Brasil. Ministério da Saúde (MS). Departamento de Análise de Situação de Saúde. Vigilância de Fatores de Risco e Proteção para Doenças Crônicas por Inquérito Telefônico, Vigitel 2009. Brasília, DF: MS; 2010.

11. Azevedo e Silva G, Valente JG, Malta DC. Trends in smoking among the adult population in Brazilian capitals: a data analysis of telephone surveys from 2006 to 2009. Rev Bras Epidemiol 2011; 14(Supl. 1):103-114.

12. Iser BPM, Claro RM, de Moura EC, Malta DC, Morais Neto OL. Risk and protection factors for chronic non communicable diseases by telephone survey -VIGITEL-2009. Rev Bras Epidemiol 2011; 14(Supl. 1):90-102.

13. Malta DC, Moura EC, de Morais Neto OL. Gender and schooling inequalities in risk and protective factors for chronic diseases among Brazilian adults, through telephone survey. Rev Bras Epidemiol 2011; 14(Supl. 1):125-135.

14. Florindo AA, Hallal PC, Moura EC, Malta DC. Practice of physical activities and associated factors in adults, Brazil, 2006. Rev Saude Publica 2009; 43(Supl. 2):65-73.
15. Malta DC, Moura EC, Silva SA, Oliveira PP, Silva VL. Prevalence of smoking among adults residing in the Federal District of Brasilia and in the state capitals of Brazil, 2008. J Bras Pneumol 2010; 36(1):75-83.

16. Francisco PM, Barros MB, Segri NJ, Alves MC, Cesar CL, Malta DC. Comparison of estimates for the self-reported chronic conditions among household survey and telephone survey-Campinas (SP), Brazil. Rev Bras Epidemiol 2011; 14(Supl. 1):5-15.

17. Brasil. Ministério do Planejamento, Orçamento e Gestão. Instituto Brasileiro de Geografia e Estatística - IBGE. Pesquisa Nacional por amostra de domicílios (PNAD 2008), Um panorama da Saúde no Brasil: acesso e utilização dos serviços, condições de saúde e fatores de risco e proteção à saúde. Rio de Janeiro: IBGE; 2010.

18. Monteiro CA, Cavalcante TM, Moura EC, Claro RM, Szwarcwald CL. Population-based evidence of a strong decline in the prevalence of smokers in Brazil (1989-2003). Bull World Health Organ 2007; 85(7): 527-534.

19. Pesquisa de Orçamentos Familiares (POF). Pesquisa de Orçamentos Familiares 2008-2009. Antropometria e Estado Nutricional de Crianças, Adolescentes e Adultos no Brasil. Rio de Janeiro, Brasília: IBGE, Ministério da Saúde; 2010.

20. World Health Organization (WHO). Prevenção de doenças crônicas, um investimento vital. Brasília: Organização Panamericana de Saúde; 2005.

21. World Health Organization (WHO).Global strategy on diet, physical activity and health. Geneva: WHO; 2004.

22. Pesquisa de Orçamentos Familiares (POF). Pesquisa de Orçamentos Familiares 2008-2009. Análise do Consumo Alimentar Pessoal no Brasil. Rio de Janeiro, Brasília: IBGE, Ministério da Saúde; 2011.

23. Jaime PC, Monteiro CA. Fruit and vegetable intake by Brazilian adults, 2003. Cad Saude Publica 2005; 21(Supl.):19-24.

24. Souza Ade M, Bezerra IN, Cunha DB, Sichieri R. Evaluation of food intake markers in the Brazilian surveillance system for chronic diseases -VIGITEL (2007-2009). Rev Bras Epidemiol 2011; 14(Supl. 1):4452.

25. Claro RM, Monteiro CA. Family income, food prices, and household purchases of fruits and vegetables in Brazil. Rev Saude Publica 2010; 44(6):10141020.

26. Jaime PC, Figueiredo IC, Moura EC, Malta DC. Factors associated with fruit and vegetable consumption in Brazil, 2006. Rev Saude Publica 2009; 43(Supl. 2):57-64.

27. Lock K, Pomerleau J, Causer L, Altmann DR, McKee M. The global burden of disease attributable to low consumption of fruit and vegetables: implications for the global strategy on diet. Bull World Health Organ 2005; 83(2):100-108.

28. Melendez JGV, Mendes LL, Pessoa MC, Sardinha LMV, Yokota RTC, Bernal RTI, Malta DC. Tendências da freqüência do consumo de feijão por meio de inquérito telefônico nas capitais brasileiras, 2006 a 2009. Cien Saude Colet. No prelo 2011. 
29. Hallal PC, Knuth AG, Reis RS, Rombaldi AJ, Malta DC, Iser BPM, Bernal RTI, Florindo AA. Time trends of physical activity in Brazil (2006-2009). Rev Bras Epidemiol 2011; 14(Supl. 1):53-60.

30. Azevedo MR, Araujo CL, Reichert FF, Siqueira FV, da Silva MC, Hallal PC. Gender differences in leisure-time physical activity. Int J Public Health 2007; 52(1):8-15.

31. Dias-da-Costa JS, Hallal PC, Wells JC, Daltoe T, Fuchs SC, Menezes AM, Olinto MTA. Epidemiology of leisure-time physical activity: a populationbased study in southern Brazil. Cad Saude Publica 2005; 21(1):275-282.

32. World Health Organization (WHO). International guide for monitoring alcohol consumption and related harm. Geneva: WHO; 2002.

33. Moura EC, Malta DC. Alcoholic beverage consumption among adults: sociodemographic characteristics and trends. Rev Bras Epidemiol 2011; 14(Supl. 1):61-70.

34. Centers for Disease Control and Prevention. Behavioral Risk Factor Surveillance System. Prevalence and Trends Data. All States 2010: Centers for Disease Control and Prevention; 2010.

35. Primer Reporte de Vigilancia de Enfermedades No Transmisibles (ENT). Situación Epidemiologica de las ENT y Lesiones en Argentina, Brasil, Chile, Paraguay y Uruguay. Santiago: Mercosur y Estados Asociados; 2011.

36. Costa JS, Silveira MF, Gazalle FK, Oliveira SS, Hallal PC, Menezes AM, Gigante DP, Olinto MT, Macedo S. Heavy alcohol consumption and associated factors: a population-based study. Rev Saude Publica 2004; 38(2):284-291.

37. Lima-Costa MF. A escolaridade afeta, igualmente, comportamentos prejudiciais à saúde de idosos e adultos mais jovens?: Inquérito de Saúde da Região Metropolitana de Belo Horizonte, Minas Gerais, Brasil. 2004. Epidemiol. Serv. Saúde 2004; 13(4):201208.

38. Almeida-Filho N, Lessa I, Magalh es L, Araujo MJ, Aquino E, Kawachi I, James SA. Alcohol drinking patterns by gender, ethnicity, and social class in Bahia, Brazil. Rev Saude Publica 2004; 38(1):45-54.

39. Barros MB, Botega NJ, Dalgalarrondo P, MarinLeon L, de Oliveira HB. Prevalence of alcohol abuse and associated factors in a population-based study. Rev Saude Publica 2007; 41(4):502-509.

40. Lima-Costa MF, Peixoto SV, Cesar CC, Malta DC, Moura EC. Health behaviors among older adults with hypertension, Brazil, 2006. Rev Saude Publica 2009; 43(Supl. 2):18-26.

41. Theme-Filha MM, Szwarcwald CL, Souza-Junior PR. Socio-demographic characteristics, treatment coverage, and self-rated health of individuals who reported six chronic diseases in Brazil, 2003. Cad Saude Publica 2005; 21(Supl):43-53.

42. Ferreira SR, Moura EC, Malta DC, Sarno F. Frequency of arterial hypertension and associated factors: Brazil, 2006. Rev Saude Publica 2009; 43(Supl. 2):98-106
43. Lima-Costa MF, Peixoto SV, Firmo JO, Uchoa E. Validity of self-reported diabetes and its determinants: evidences from the Bambui study. Rev Saude Publica 2007; 41(6):947-953.

44. Barros MBdA, Galvão CL, Carandina L, Torre GD. Social inequalities in the prevalence of chronic diseases in Brazil, PNAD 2003. Cienc Saude Colet 2006; 11(4):911-926.

45. Instituto Nacional do Câncer (INCA). Inquérito Domiciliar sobre Comportamentos de Risco e Morbidade Referida de Doenças e Agravos não Transmissíveis. Brasil, 15 capitais e Distrito Federal 2002-2003. Rio de Janeiro: INCA; 2004.

46. Danaei G, Finucane MM, Lin JK, Singh GM, Paciorek CJ, Cowan MJ, Farzadfar F, Stevens GA, Lim SS, Riley LM, Ezzati M. National, regional, and global trends in systolic blood pressure since 1980: systematic analysis of health examination surveys and epidemiological studies with 786 country-years and 5.4 million participants. Lancet 2011; 377(9765):568577.

47. Danaei G, Finucane MM, Lu Y, Singh GM, Cowan MJ, Paciorek CJ, Lin JK, Farzadfar F, Khang YH, Stevens GA, Rao M, Ali MK, Riley LM, Robinson CA, Ezzati M. National, regional, and global trends in fasting plasma glucose and diabetes prevalence since 1980: systematic analysis of health examination surveys and epidemiological studies with 370 country-years and 2.7 million participants. Lancet 2011; 378(9785):31-40.

48. Barreto SM, Figueiredo RC. Chronic diseases, selfperceived health status and health risk behaviors: gender differences. Rev Saude Publica 2009; 43(Supl. 2):38-47.

49. Schmidt MI, Duncan BB, Hoffmann JF, Moura L, Malta DC, Carvalho RM. Prevalence of diabetes and hypertension based on self-reported morbidity survey, Brazil, 2006. Rev Saude Publica 2009; 43(Supl. 2):74-82.

50. Malerbi DA, Franco LJ. Multicenter study of the prevalence of diabetes mellitus and impaired glucose tolerance in the urban Brazilian population aged 30-69 yr. The Brazilian Cooperative Group on the Study of Diabetes Prevalence. Diabetes Care 1992; 15(11):1509-1516.

51. Yokota RTC, Iser BPM, Andrade RLM, Santos Jd, Meiners MMMA, Assis DM, Bernal RTI, Malta DC, Moura L, Santos J. Vigilância de fatores de risco e proteção para doenças e agravos não transmissíveis em município de pequeno porte, Brasil, 2010. Epidemiol. Serv. Saúde 2012; 21(1):55-68.

52. Neves AP, Wehrmeister FC, Martínez-Mesa J, Cascaes AM, Menezes AM. Tendência temporal de asma em idosos brasileiros: análise da Pesquisa Nacional por Amostra de Domicílios (PNAD). In: XX Congresso de Iniciação Científica, Universidade Federal de Pelotas; 2011; Pelotas.

53. Cooper PJ, Rodrigues LC, Cruz AA, Barreto ML. Asthma in Latin America: a public heath challenge and research opportunity. Allergy 2009; 64(1):5-17. 
54. Wehrmeister FC, Menezes AM, Cascaes AM, Martinez-Mesa J, Barros AJ. Time trend of asthma in children and adolescents in Brazil, 1998-2008. Rev Saude Publica; 46(2):242-250.

55. Viacava F. Acesso e uso de serviços de saúde pelos brasileiros. RADIS 2010; 96:12-19.

56. Barros MB, Zanchetta LM, Moura EC, Malta DC. Self-rated health and associated factors, Brazil, 2006. Rev Saude Publica 2009; 43(Supl. 2):27-37.

57. Lima-Costa MF, Loyola Filho AI, Matos DL. Trends in health conditions and use of health services by the Brazilian elderly: a study based on the National Household Sample Survey $(1998,2003)$. Cad Saude Publica 2007; 23(10):2467-2478.

58. Instituto Nacional do Câncer (INCA). Incidência de Câncer no Brasil, Estimativa 2012. Rio de Janeiro: INCA; 2012.

59. Szklo AS, Almeida LM, Figueiredo V, Lozana J de A, Azevedo e Silva Mendonca G, Moura L, Szklo M. Behaviors related to sunlight exposure versus protection in a random population sample from 15 Brazilian State capitals and the Federal District, 20022003. Cad Saude Publica 2007; 23(4):823-834.

60. Rizzatti K, Schneider IJC, d'Orsi E. Perfil epidemiológico dos cidadãos de Florianópolis quanto à exposição solar. Epidemiol. Serv. Saúde 2011; 20(4): 459-469.

61. Castilho IG, Sousa MA, Leite RM. Photoexposure and risk factors for skin cancer: an evaluation of behaviors and knowledge among university students. An Bras Dermatol 2010; 85(2):173-178.

62. Okura Y, Urban LH, Mahoney DW, Jacobsen SJ, Rodeheffer RJ. Agreement between self-report questionnaires and medical record data was substantial for diabetes, hypertension, myocardial infarction and stroke but not for heart failure. J Clin Epidemiol 2004; 57(10):1096-1103.

63. Mokdad AH, Remington PL. Measuring health behaviors in populations. Prev Chronic Dis 2010; 7(4):A75.

Artigo apresentado em 10/05/2012

Aprovado em 22/06/2012

Versão final apresentada em 29/07/2012 